\title{
Cerebral Lateralization of Frontal Lobe Language Processes and Lateralization of the Posterior Visual Word Processing System
}

\author{
Qing Cai ${ }^{1}$, Michal Lavidor ${ }^{2}$, Marc Brysbaert ${ }^{3}$, Yves Paulignan ${ }^{1}$, \\ and Tatjana A. Nazir ${ }^{1}$
}

\begin{abstract}
The brain areas involved in visual word processing rapidly become lateralized to the left cerebral hemisphere. It is often assumed this is because, in the vast majority of people, cortical structures underlying language production are lateralized to the left hemisphere. An alternative hypothesis, however, might be that the early stages of visual word processing are lateralized to the left hemisphere because of intrinsic hemispheric differences in processing low-level visual information as required for distinguishing fine-grained visual forms such as letters. If the alternative hypothesis was correct, we would expect posterior occipito-temporal processing stages still to
\end{abstract}

\section{INTRODUCTION}

Despite substantial limits imposed by our visual system, skilled reader can identify words of more than 10 characters within a single glance in a fraction of a second (Erdmann \& Dodge, 1898) —at least if these words are presented in a familiar format in central vision or to the right of fixation (Nazir, Ben-Boutayab, Decoppet, Deutsch, \& Frost, 2004; Young \& Ellis, 1985). Over the last decade, brain imaging studies have provided insights into the cortical structures that are involved in this skill, and one of the most striking findings is the rapid lateralization of these structures to the left hemisphere (LH). Cohen et al. (2000) were among the first to suggest that a localized region in the left fusiform cortex played a key role in visual word processing by extracting an invariant orthographic representation from the visual input. They called this region the "visual word form area (VWFA)" (see also Cohen \& Dehaene, 2004; McCandliss, Cohen, \& Dehaene, 2003; Price \& Devlin, 2003) and subsequent research indeed showed that this region is more responsive to orthographic stimuli than to digits (Polk et al., 2002) or to geometric forms (Gros, Doyon, Rioual, \& Celsis, 2002), to words than to nonwords (Vigneau,

\footnotetext{
${ }^{1}$ Institut des Sciences Cognitives, Bron Cedex, France, ${ }^{2}$ University of Hull, UK, ${ }^{3}$ University of London, Egham, Surrey, UK
}

be lateralized to the left hemisphere for participants with right hemisphere dominance for the frontal lobe processes involved in language production. By analyzing event-related potentials of native readers of French with either left hemisphere or right hemisphere dominance for language production (determined using a verb generation task), we were able to show that the posterior occipito-temporal areas involved in visual word processing are lateralized to the same hemisphere as language production. This finding could suggest top-down influences in the development of posterior visual word processing areas.

Jobard, Mazoyer, \& Tzourio-Mazoyer, 2005), and is activated regardless of letter case or font, or of stimulus location in the visual field (Dehaene et al., 2004; Cohen et al., 2000).

Other brain areas surrounding the VWFA have similarly been found to be left lateralized. A particularly relevant study was recently run by Gold and Rastle (2007), who made use of the finding that in a functional magnetic resonance imaging (fMRI) study the blood oxygenation level-dependent response to a word is smaller if the word has been preceded by a related prime than when it has been preceded by an unrelated prime. Using a variety of different primes, these authors could identified three left lateralized occipito-temporal regions that seemed to support a purely structural morphemic segmentation mechanism, which operates independently of semantic information at early stages of visual word processing. Two of these regions also showed orthographic priming (i.e., a posterior portion of the left fusiform gyrus, including the VWFA and an extrastriate region in a posterior portion of the middle occipital gyrus), whereas the third region (an anterior portion of the middle occipital gyrus) was sensitive only to morphological similarity between primes and targets. Brain regions sensitive to semantic similarity were clearly distinct from those sensitive to morphological structure (semantic priming was observed in a portion of the left middle temporal gyrus). Important for 
us was again the finding that all these structures were strongly lateralized to the left.

The question we addressed in the present study was why visual word processing lateralizes already in occipitotemporal areas. Usually, it is assumed this is because the LH for most people is the "language-dominant" hemisphere. However, this explanation overlooks the facts that cerebral dominance usually refers to the laterality of language production controlled by structures in the frontal lobes (in particular, Broca's area and the surrounding regions), whereas reading and writing are relatively recent human skills making use of structures in the occipitotemporal cortex that were not designed for reading purposes (Dehaene, Cohen, Sigman, \& Vinckier, 2005). Moreover, already from the very first split-brain studies, it was clear that laterality of language production did not imply lateralization of meaning because patients could indicate with their left hand which object had been presented to the right hemisphere ( $\mathrm{RH})$, even though they could not name it (e.g., Gazzaniga, 1983).

It is reasonable to assume that the functional role of posterior visual word processing areas develops with reading practice (see Brem et al., 2006; Maurer, Brem, Bucher, \& Brandeis, 2005). Because visual word recognition consists of a visual and a linguistic component, the lateralization of these functions could thus have two origins. Either the posterior visual word processing system develops primarily in the left cerebral hemisphere because of better interactions between this part of the brain and the structures responsible for speech functions, or the system lateralizes to the LH because this hemisphere is more efficient in processing low-level visual information required for distinguishing fine-grained forms in print.

Given the extensive visual training that comes with reading, the specialization of the left occipito-temporal region for processing print could result from alterations in purely visual processes underlying visual word recognition. Electrophysiological and fMRI evidence for the emergence of domain-specific visual expertise in occipitotemporal object-related brain regions has, indeed, been demonstrated for several classes of stimuli, including pictures of birds, dogs (Tanaka \& Curran, 2001), cars (Gauthier, Curran, Curby, \& Collins, 2003), buildings, faces, sculptures (Grill-Spector, Sayres, \& Ress, 2006; Malach, Levy, \& Hasson, 2002), chairs (Ishai, Ungerleider, Martin, Schouten, \& Haxby, 1999), and fingerprints (Busey \& Vanderkolk, 2005). According to Malach et al. (2002), these higher-order cortical object areas form a natural extension of the early retinotopic cortex, and principles of the topographic organization of the early visual cortex extend into these regions in that they also contain an orderly map of eccentricity. The topography of object representations within these regions is basically shaped bottom-up by acuity demands during acquisition. That is, processes that require fine detail analyses will be processed initially in higher-order cortical object areas that receive strong input from high-resolution foveal representations. By contrast, processes that depend on large-scale integration will be processed in cortical regions that receive input from peripheral representations. Following this view, the visual word processing areas in the left occipito-temporal region supply functions common to visual objects processing in general and are not specific to linguistic stimuli (for related proposals, see Devlin, Jamison, Gonnerman, \& Matthews, 2006; Xue, Chen, Jin, \& Dong, 2006; Price \& Friston, 2005). They just grew out of the fact that the left visual cortex might be more efficient at processing fine-grained visual information.

The alternative view is that the posterior occipitaltemporal areas evolve and develop as part of the left lateralized cortical structures that support language. In this view, the laterality of language production would have modulating effects on the laterality of other language functions, including those related to reading (Hillis et al., 2005; Kosslyn, 1987). In most individuals, the hemisphere, which is dominant for speech functions, is the LH (Szaflarski et al., 2002; Knecht et al., 2000; Pujol, Deus, Losilla, \& Capdevila, 1999). Because the elaboration of phonological and orthographic word forms may be conditional upon easy access to this system (Hillis et al., 2005), the bias of the posterior word recognition system toward the $\mathrm{LH}$ could indicate that it develops as part of a wider cortical language network. Unlike the previous hypothesis, the present assumption entails that the development of the visual word processing system is controlled top-down by requirements of cortical language structures in the frontal lobe and is thus specific to the processing of linguistic stimuli (for a related proposal, see Hillis et al., 2005).

In the present study, we examine what happens when language production is lateralized to the $\mathrm{RH}$. We do so by looking at the laterality of the posterior word recognition areas in French readers with $\mathrm{RH}$ dominance for frontal language processes (see Hunter, Brysbaert, \& Knecht, 2007 and Brysbaert, 1994, for a similar approach).

Although for the vast majority of individuals language production lateralizes to the $\mathrm{LH}$, a minority (some $5 \%$ of the right-handers and some $30 \%$ of the left-handers) has either reversed laterality or bilateral representation. In addition, nowadays we are able to assess the laterality of language production in a valid and noninvasive way. The techniques include functional transcranial Doppler sonography (fTCD; e.g., Hunter et al., 2007; Knecht et al., 2001), fMRI (e.g., Hunter et al., 2007; Rowan et al., 2004; Szaflarski et al., 2002; Knecht et al., 2001; Pujol et al., 1999) and evoked response potentials (ERPs; e.g., Rowan et al., 2004). Using fTCD and fMRI, Knecht et al. (2000) provided evidence that $4 \%$ of a randomly selected sample of strong right-handers were RH-dominant for language production, against $27 \%$ of the strong lefthanders. Based on these findings, we invited students who scored high for either left- or right-handedness and 
assessed their brain dominance during a verb generation task, which is known to reveal robust lateralizing effects in the inferior frontal cortex (Thiel et al., 1998; Frith, Friston, Liddle, \& Frackowiak, 1991; Petersen, Fox, Posner, Mintun, \& Raichle, 1988). A selection of these students then took part in a further study on the laterality of the occipito-temporal word recognition regions.

To assess laterality of language production, we replicated an ERP study by Rowan et al. (2004). These authors presented nouns to their participants (e.g., broom) and asked them to generate a verb that could be used with the noun (e.g., sweep). They observed an anterior slow cortical potential related to the verb generation task, which took the form of a late sustained negativity over inferior frontal regions and which was stronger over the dominant hemisphere as identified with fMRI (see also Thomas, Altenmuller, Marckmann, Kahrs, \& Dichgans, 1997).

To measure the laterality of the posterior visual word processing system, we replicated an ERP study by Cohen et al. (2000). These authors used fMRI and ERP to reveal the early processing stages of parafoveally displayed visual words. They first observed activation in the occipital cortex contralateral to the stimulated field. They then observed increased activation in the left inferior temporal electrodes, which was identical for right visual field (RVF) and left visual field (LVF) stimulation. The signal consisted of a unilateral sharp negativity, recorded around 200 msec poststimulus. The origin of the signal (the VWFA in the left middle portion of the fusiform gyrus) was established by fMRI data (see also Brem et al., 2006).

Our hypothesis is straightforward. If the hemispheric asymmetry in processing low-level information is responsible for the laterality of the posterior visual word processing system, we should observe left laterality independently of the participants' lateralization of frontal lobe language functions as indexed in the verb generation task. In contrast, if the laterality of this system is determined by frontal language structures, then we should observe a perfect correlation between the laterality measured in the verb generation task and the laterality measured in the parafoveal word reading task.

\section{METHODS}

\section{Participants}

Fifteen right-handed and 11 left-handed participants took part in the study. Handedness was defined using the Edinburgh handedness questionnaire (Oldfield, 1971) (right-handers scored $>0.5$, mean score $=0.9$; left-handers scored $<-0.5$ with one exception scored $=-0.1$, mean score of left-handers $=-0.6)$. All participants were students at the university in Lyon (19-38 years) and were native speakers of French with no neurological or psy- chiatric history. All had normal or corrected-to-normal vision. Following a number of selection criteria that will be described later, eight participants with LH dominance for language production and four participants with $\mathrm{RH}$ dominance for language production were retained.

\section{Verb Generation}

Stimuli

Stimuli consisted of 80 concrete nouns, with an average $\log _{10}$ frequency/million $=1.37$ (Frant frequency; New, Pallier, Ferrand, \& Matos, 2001) and an average length of 6.11 letters (range between 4 and 9 letters).

\section{Design and Procedure}

The design of this experiment was kept as close as possible to the design used by Rowan et al. (2004), with the exception that, in our study, nouns were presented visually, whereas Rowan et al. presented their stimuli orally. Participants were instructed to silently generate a single verb in relation to the visually presented noun. A trial began with the presentation of a central cross-hair acting serving as visual fixation point. Participants were instructed to maintain their gaze on the fixation cross until the end of the trial. After $2000 \mathrm{msec}$, the cross-hair was replaced by a noun, which was presented centrally on the screen and remained visible for $500 \mathrm{msec}$. With the offset of the stimulus, the cross-hair reappeared. After $2500 \mathrm{msec}$, two vertical lines appeared above and below the cross-hair, prompting participants to pronounce the generated verb. This overt component was included to ensure that the task was being performed. A digital video was used to record participants' response.

\section{Data Acquisition}

Electroencephalogram (EEG) data were recorded continuously from 32 electrodes referenced against $\mathrm{Cz}$. Horizontal and vertical electrooculograms (EOG) were recorded from bipolar electrodes placed on the left outer canthus (hEOG) and below the left eye (vEOG). ERPs were sample at $2500 \mathrm{~Hz}$, and system band pass was 0.016-1000 Hz. Impedances were kept $<10 \mathrm{k} \Omega$ throughout the experiments. Thirty-two sintered $\mathrm{Ag} / \mathrm{AgCl}$ ring electrodes were placed on elastic caps (ECI Electro-Cap, Electro-Cap International, USA) positioned according to the international 10-20 system (BrainAmp MR, Brain Products GmbH, Munich, Germany) matched to the head size of each participant.

\section{Data Analyses}

ERP data were processed using BrainAmp analyse software. Data were band-pass filtered (0.016-40 Hz, $48 \mathrm{~dB} /$ oct) and re-referenced to a common average reference 
including Cz. Data were segmented from 200 msec prior to stimulus onset to $3000 \mathrm{msec}$ post onset. Artifact rejection was done automatically by rejecting trials with potential exceeding $\pm 150 \mu \mathrm{V}$ or transients exceeding $\pm 50 \mu \mathrm{V}$ between two samples. Trials with obvious effects of eye movements and blinking were excluded manually. The remaining trials were corrected for baseline over a 200-msec window prior to stimulus onset. Data were averaged for each individual. ERP mean amplitudes of the electrodes over left and right inferior frontal regions were calculated and compared. Stronger late sustained slow cortical potential negativity within a time window of 1250-2800 msec at electrodes over left than over right inferior frontal regions was taken as selection criterion for LH language dominance and vice versa. Analyses of variances (ANOVAs) were used to compare potentials on the homologue electrodes for the two groups.

\section{Parafoveal Word Reading}

Stimuli

Stimuli were 50 words and 50 consonants strings. All stimuli were five letters long. The words had one or two syllables, and had an average $\log _{10}$ frequency/million $=1.54$ (range between 0 and 2.4; Frant frequency). All stimuli were presented once in the RVF and once in the LVF.

\section{Design and Procedure}

The design of this experiment was kept as close as possible to the design used by Cohen et al. (2000). In each trial, participants were asked to fixate a permanent central cross-hair while words or consonant strings were briefly displayed in their RVF or LVF. Stimulus eccentricity was $3.6^{\circ}$ to the center of the stimulus and $5.8^{\circ}$ to the outer edge of stimulus (visual angle $1.4^{\circ}-5.8^{\circ}$ ) in either visual field. Five hundred milliseconds following the onset of the cross-hair, the stimulus was displayed for $150 \mathrm{msec}$. One thousand eight hundred milliseconds after stimulus offset, two vertical lines appeared above and below the cross-hair, prompting participants to name the word or to say "rien" (nothing) upon seeing a nonword. Trials were self-paced, and stimuli were shuffled randomly. A digital video was used to record participants' response. Consonant strings were used as nonwords to produce a big difference between the word and the nonword trials. Participants were asked to name the word or to say "rien" (mean "nothing") for nonwords to make sure they really read the words, but a time delay was introduced to avoid/minimize artifact contamination (e.g., from muscles) in the EEG data.

\section{Data Acquisition}

Data acquisition was identical to the verb generation task.

\section{Data Analyses}

Data were band-pass filtered (0.5-20 Hz), re-referenced, and segmented from $200 \mathrm{msec}$ prior to stimulus onset to $2000 \mathrm{msec}$ poststimulus. Artifact rejection was executed following the same parameters used in the verb generation task. To sharpen the data and further analyze local brain activity, potential distributions were interpolated on a sphere using spherical splines (order of splines $=4$, maximum degree of Legendre polynomials $=10$ ), current source densities (CSDs) were estimated for all recording sites. Compared to raw EEG data, CSD is reference-free, that is, this analysis allows calculating activity at critical electrodes independently of the activity at the reference electrode. Moreover, CSD transformation has also the property to act as a spatial high-pass filter, and therefore, it enhances the contribution of local brain activity to the signal, while global contributions of distant sources are minimized (Pulvermüller, Lutzenberger, \& Preissl, 1999; Law, Rohrbaugh, Adams, \& Eckardt, 1993; Perrin, Bertrand, \& Pernier, 1987). This property can be beneficial for asymmetry studies because activity of one hemisphere/ region that is introduced via volume conduction to the other hemisphere/region would be considerably reduced with a CSD transform (Hagemann, 2004). Data for word presented in the LVF and RVF were averaged separately for each individual. Grand mean averages were calculated separately for LH and RH language-dominant participants. 2-D maps of scalp potential were constructed for the same time window. To compare the evoked current densities on homologous inferior temporal electrodes, ANOVAs were conducted on two time windows of interests.

\section{RESULTS}

The data of five right-handed and two left-handed participants could not be used because they failed to reach impedance $<10 \mathrm{k} \Omega$, they had high noise-to-signal ratio, or they failed to fixate at the central cross-hair in the parafoveal word reading task. This left us with 10 righthanded and 9 left-handed participants.

\section{Verb Generation}

For each participant, the potentials recorded over the left inferior frontal region within the critical time window (1250-2800 msec after word onset) were subtracted from those recorded over the right inferior frontal region. Negative scores thus indicate a stronger negativity over the LH and positive scores indicate a stronger negativity over the RH. Figure 1A shows the subtraction scores averaged over the entire time window separately for the 10 right-handers and the 9 left-handers. Eight of the right-handers had a laterality index larger than $+0.5 \mu \mathrm{V}$ and were selected as LH-dominant for language production; four of the lefthanders had an index smaller than $-0.5 \mu \mathrm{V}$ and were selected as RH-dominant for language production. 
Figure 1. (A) ERP subtraction scores of homologous electrodes over the left and right inferior frontal regions for individual participants during verb generation. Data are averaged over a time window of 1250-2800 msec after word onset. Negative scores indicate a stronger negativity over the LH and positive scores a stronger negativity over the RH. Eight of the right-handers and four of the left-handers were considered fulfilling the selection criterion which we set to $> \pm 0.5 \mu \mathrm{V}$. These participants were considered LH- and RH-dominant for language production, respectively. (B). ERPs recorded on homologous electrodes over the left and right inferior frontal regions during verb generation and ERP topography in the time window of 1250-2800 msec poststimulation, averaged for LH- and RH-dominant participants, respectively. ERP traces are shown from $200 \mathrm{msec}$ before presentation of the stimulus noun $(0 \mathrm{msec})$ to 2800 msec poststimulation.

\section{A}
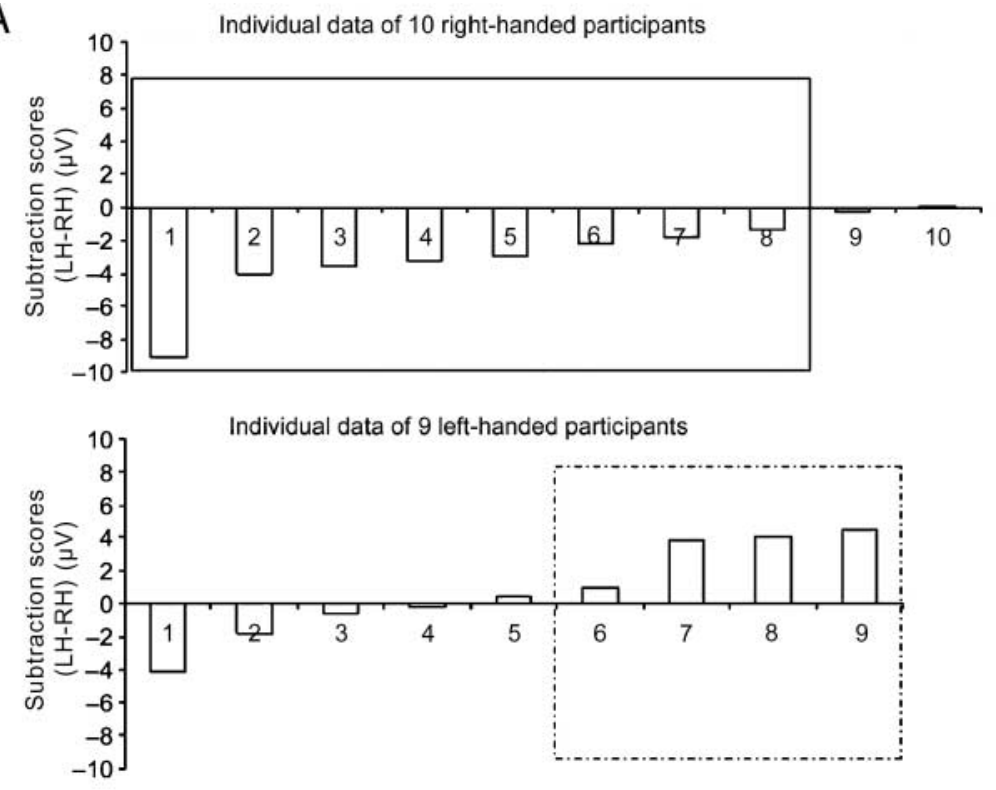

B Averaged data of $8 \mathrm{LH}$-dominant participants during verb generation
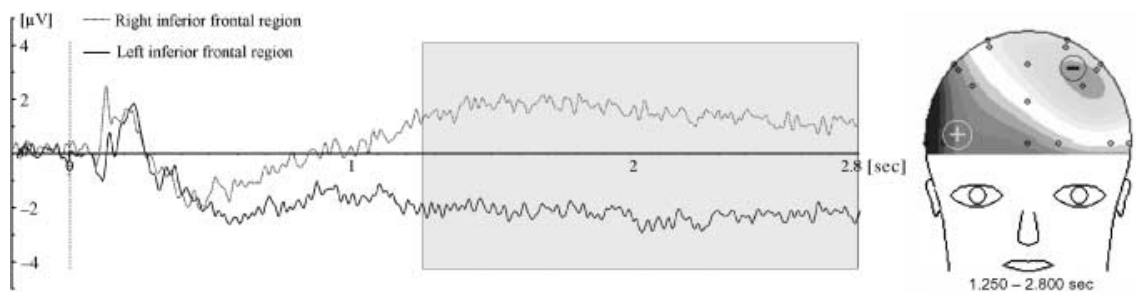

Averaged data of $4 \mathrm{RH}$-dominant participants during verb generation

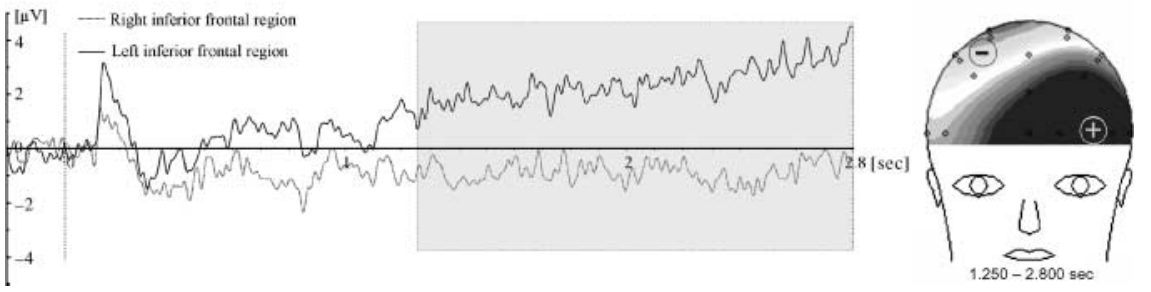

Figure 1B plots ERP group-average for the selected eight right-handers and four left-handers for homologue electrodes over the left and right inferior frontal regions. An ANOVA performed for the critical time window showed that potentials recorded over the homologue regions were significantly different in both groups $[F(1,7)=16.81$, $p<.01$ and $F(1,3)=17.06, p=.03$, for LH-dominant and RH-dominant participants, respectively].

\section{Parafoveal Word Reading}

Behavioral Results for the $\mathrm{LH}$ - and RH-dominant Participants

All LH-dominant participants showed a strong RVF advantage. Average correct percentage was $93.0 \%$ for words displayed in the RVF and $69.3 \%$ for words displayed in the $\operatorname{LVF}[F(1,7)=30.85, p=.001]$. Performance for the four RH-dominant participants were high and did not show a reliable difference between the visual fields [93.8\% for words displayed in the RVF and 91.5\% for words displayed in the LVF; $F(1,3)=0.49, p=$ $.54]$.

\section{ERP Results}

Figure 2 shows the averaged potentials recorded at homologous right and left inferior temporal electrodes during presentation of words in the RVF and LVF. Data are given separately for LH- and RH-dominant participants. The topography of the N1 wave is shown for two critical time windows corresponding to the early negativity 
Figure 2. (A) Averaged data of LH-dominant participants in the parafoveal word reading task. (B) Averaged data of RH-dominant participants. Upper panel shows ERP group average recorded on the left and right inferior temporal electrodes during the presentation of words in the LVF and in the RVF (strictly it is more appropriate to speak about "evoked current source densities [CSDs] "); lower panel shows ERP topography in an early time window (120-155 msec, surrounding the onset of N1) and in a late time window (188-223 msec, surrounding the peak of N1) during the presentation of words in the LVF and RVF. Red $=$ positive , green $=$ negative. Arrow indicates the lateralization of the visual word processing system.

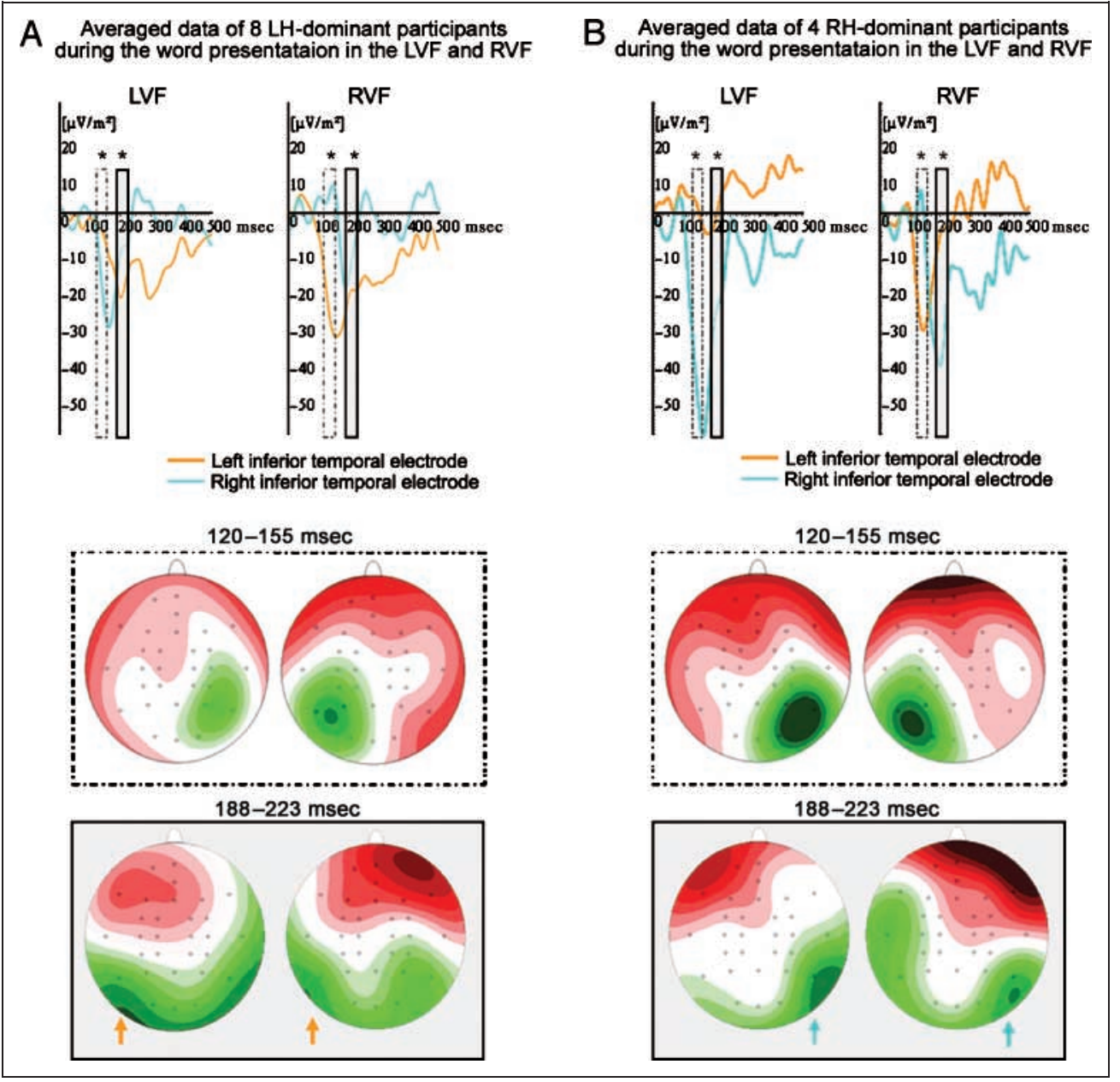
during the word presentataion in the LVF and RVF

$120-155 \mathrm{msec}$

$188-223 \mathrm{msec}$
A Averaged data of $4 \mathrm{RH}$-dominant participants Aving the word presentataion in the LVF and RVF

$188-223 \mathrm{msec}$ surrounding the onset of the N1 component and the later negativity surrounding the peak of the N1 component, respectively.

LH-dominant participants. The results of LH-dominant participants replicate the findings reported by Cohen et al. (2000). About $150 \mathrm{msec}$ after word onset, an early negative waveform (N1) is visible as a posterior negativity contralateral to the stimulated visual field. A two-factor repeated measures ANOVA (visual field and hemisphere) on the inferior temporal electrodes with potentials averaged over the 35-msec time window surrounding the onset of the N1 (120-155 msec) showed no significant overall effect of visual filed $[F(1,7)=0.08, p=.79]$, a significant effect of hemisphere $[F(1,7)=9.43, p=.02]$, and a significant interaction between the two variables $[F(1,7)=37.87, p<.001]$. Newman-Keuls post hoc analysis showed that the potentials were significantly more negative over the contralateral hemisphere both for words presented in the $\operatorname{RVF}(p<.01)$ and words presented in the LVF $(p=.04)$.

In line with Cohen et al.'s study, at around $200 \mathrm{msec}$ poststimulation onset, the waveforms became similar for LVF and RVF displays. A two-factor ANOVA on the same two electrodes with potentials averaged over the 35-msec time window surrounding the peak of N1 component (188-223 msec) showed no effect of visual field $[F(1,7)=0.94, p=.37]$, whereas the effect of hemisphere was significant $[F(1,7)=17.28, p<.01]$, that is, a significantly stronger negativity was observed over the left inferior temporal electrode than over the homologous right electrode independently of where in the visual field the words were presented.

RH-dominant participants. The data for RH-dominant participants showed a very similar initial pattern of results for the onset of the N1 component (see Figure 2). A two-factor ANOVA on inferior temporal electrodes with potentials averaged over a 35-msec time window (120$155 \mathrm{msec}$ ) showed no significant overall effect of visual field $[F(1,3)=0.18, p=.70]$ or hemisphere $[F(1,3)=$ $0.53, p=.52]$, but a significant interaction between the two factors $[F(1,3)=48.08, p<.01]$. Post hoc analysis showed that potentials were significant more negative on the left inferior temporal electrode than on right inferior temporal electrode for RVF displays $(p=.03)$, 
and the reversed pattern was observed for LVF displays $(p=.03)$.

In the time window surrounding the peak of the N1 component (188-223 msec), a two-factor ANOVA on the same two electrodes showed no effect of visual field $[F(1,3)=1.14, p=.36]$ but a significant effect of hemisphere $[F(1,3)=50.69, p<.01]$. In contrast to LHdominant participants, for RH-dominant participants the negativity was stronger over the right inferior temporal electrode. Individual data for RH-dominant participants (Table 1) show that the reported effects were consistent over all participants.

\section{DISCUSSION}

In the present study, we examined the laterality of the occipito-temporal visual word recognition areas in French speakers who had been assessed as left-dominant or rightdominant for frontal lobe language processes in a verb generation task. We hypothesized that if the laterality of these areas is due to the hemispheric asymmetry in processing low-level visual information, we should observe a left laterality for both groups of participants. In contrast, if the laterality of the visual word recognition areas follows that of language production, we should find evidence for left laterality in the LH-dominant group and right laterality in the $\mathrm{RH}$ group.

Our findings were extremely clear: There was no evidence whatsoever in favor of the low-level visual pro- cessing hypothesis. There was a perfect correlation between the inferior frontal laterality index obtained in the verb generation task and the posterior occipitaltemporal laterality index obtained in the parafoveal word reading task.

Interestingly, the data were much more clear-cut for the ERP data than for the behavioral data. The RH dominance was clearly present in the EEG signal, whereas there was no clear difference in word recognition performance for the RH-dominant participants after presentation in the LVF (91.5\% of the words identified) or in the RVF (93.8\% of the words identified). Inconsistencies between VHF differences in the past have been used as an argument that different language functions may be lateralized differently (e.g., Andresen \& Marsolek, 2005; Boles, 1998). Our data suggest that part of these inconsistencies may have been due to the fact that behavioral measures (RTs, percentage correct) may not always provide a valid measure of brain laterality. However, because performance for our RH-dominant participants was close to ceiling, further studies are needed to better clarify this point.

The fact that the visual word recognition system lateralizes to the same side as structures involved in verb generation is suggestive of the assumption that the laterality of the posterior visual word processing system is a (top-down) function of the interaction with other word processing structures and not a (bottom-up) consequence of the visual input. In other words, this system may be primarily a language system and not a visual

Table 1. Individual Data of RH-dominant Participants for the Parafoveal Word Reading Task

\begin{tabular}{|c|c|c|c|c|}
\hline \multirow[b]{3}{*}{ Subject } & \multicolumn{4}{|c|}{ Evoked Current Densities $\left(\mu \mathrm{V} / \mathrm{m}^{2}\right)$} \\
\hline & \multicolumn{2}{|c|}{$L V F$} & \multicolumn{2}{|c|}{$R V F$} \\
\hline & $\begin{array}{c}\text { Right Inferior } \\
\text { Temporal Electrode }\end{array}$ & $\begin{array}{c}\text { Left Inferior } \\
\text { Temporal Electrode }\end{array}$ & $\begin{array}{c}\text { Right Inferior } \\
\text { Temporal Electrode }\end{array}$ & $\begin{array}{c}\text { Left Inferior } \\
\text { Temporal Electrode }\end{array}$ \\
\hline \multicolumn{5}{|c|}{ 120-155 msec (Surrounding the Onset of N1) } \\
\hline RH_s01 & -26.8 & 9.7 & 3.7 & -28.6 \\
\hline RH_s02 & -40.7 & -6.5 & -13.5 & -24.5 \\
\hline RH_s03 & -67.2 & -3.4 & 1.8 & -11.9 \\
\hline RH_s04 & -19.4 & 13.5 & 13.5 & -48.5 \\
\hline Average & -38.5 & 3.3 & 1.3 & -28.4 \\
\hline \multicolumn{5}{|c|}{ 188-223 msec (Surrounding the Peak of N1) } \\
\hline RH_s01 & -58.6 & -16.4 & -34.8 & -26.7 \\
\hline RH_s02 & -31.0 & -17.8 & -49.4 & -19.4 \\
\hline RH_s03 & -30.7 & -2.6 & -48.0 & -5.2 \\
\hline RH_s04 & -9.0 & 27.7 & -15.8 & 27.4 \\
\hline Average & -32.3 & -2.3 & -37.0 & -6.0 \\
\hline
\end{tabular}


processing system. Our data do not allow us to make hard claims concerning the VWFA itself, but there are strong suggestions that this structure is included within the lateralized posterior language system. First, all current evidence on the basis of unselected participant groups points to a left laterality of this structure (e.g., Hillis et al., 2005; Cohen et al., 2000). Second, the ERP signal we used in the parafoveal word reading experiment is the same signal Cohen et al. (2000) identified as the signal coming from the VWFA, and Brem et al.'s (2006) study also showed a positive correlation between fMRI activation in the VWFA and late occipito-temporal N1 amplitude for words. Finally, the early onset of the lateralization, despite the fact that the stimuli were presented parafoveally, makes us quite confident that the VWFA is located in the same hemisphere as lateralized frontal lobe structures that support language.

The idea that top-down influences from anterior cortical regions may have a decisive influence in the development of posterior stimulus processing structures has recently also been suggested for object recognition. Bar et al. (2006) provided evidence for top-down facilitation in visual object recognition. They showed that object recognition elicited activity in the orbito-frontal cortex 50 msec earlier than activation in the higher-order object areas in the temporal cortex. The early orbito-frontal activity was modulated by low spatial frequency information in the image. Based on these observations, Bar et al. suggested that the prefrontal region mediates visual object recognition via top-down connections by providing an "initial guess" about object identity. This "initial guess" reduces the set of probable interpretations of the input image in recognition related areas within the temporal cortex, which are then integrated with a bottomup analysis of the stimulus for recognition. The present data do not allow drawing direct parallels between object recognition and visual word recognition. However, it is tempting to assume that some common principles underlie the development of visual object representations and representations of printed words in occipitotemporal areas.

A note of caution has to be added though. In theory, the lateralization of anterior language processes and the lateralization of visual processes that underlies reading could be independently driven by a third variable (genetic and/or environmental; see Andresen \& Marsolek, 2005; Vallortigara \& Rogers, 2005 for review). The extreme case of this scenario would be a complete mirror reversal of the laterality of all functions in our $\mathrm{RH}$ dominant participants. Note though that people with complete situs inversus (i.e., a condition in which the organs of the chest and abdomen are arranged in a perfect mirror image reversal of the normal positioning), while showing complementary reversal of frontal and occipital petalia, do not show reversal in handedness or language dominance (see Sun \& Walsh, 2006 for a review). This suggests that organ as well as functional asymmetries might be regulated independently. The fact that in our data we observed a perfect correlation between the inferior frontal laterality index obtained in the verb generation task and the posterior occipitaltemporal laterality index obtained in the parafoveal word reading task might thus rather suggest that the two functions are part of the same network. Note also that previous studies that mapped asymmetries of the homologue of Broca's area in the brains of great apes found that the simian brains have asymmetries, which resemble those of humans. This suggests that frontal brain structures associated with language ability might have existed before humans evolved (Cantalupo \& Hopkins, 2001). Given that reading and writing are relatively recent human skills that make use of (occipito-temporal) structures, which were not designed for reading purposes (Dehaene et al., 2005), it seems unlikely that the lateralization of the posterior visual word processing system is independently driven by the same variable(s) that also drive the lateralization of frontal lobe language processes.

Comparison of conditions that trigger activity in posterior occipito-temporal regions during word recognition with those that trigger differential activation during recognition of other visual object categories might provide a method to further test the "common cause" hypothesis and to specify whether and how higher-order areas contribute to the perception of visual stimuli, and why visual processes of some stimulus categories are lateralized (e.g., faces, words) while others are not.

\section{Conclusion}

By analyzing event-related potentials of native readers of French with either LH or RH dominance for language production, we were able to show that processes within posterior occipito-temporal areas that are involved in visual word recognition lateralized to the same hemisphere as frontal lobe language processes. This result suggests that the laterality of the visual word recognition system may be a function of the interaction with other word processing structures and not a consequence of the asymmetry of low-level visual processing. We suggest that top-down influences from the inferior frontal cortex may have a decisive influence in the function and lateralization of posterior word recognition areas including VWFA.

\section{Acknowledgments}

This study was supported by a European Commission funded research and training (RTN:LAB, MRTN-CT-2004-512141) entitled Language and Brain.

Reprint requests should be sent to Qing Cai, Institut des Sciences Cognitives, 67, boulevard Pinel, 69675 Bron Cedex, France, or via e-mail: Qing.Cai@isc.cnrs.fr. 


\section{REFERENCES}

Andresen, D. R., \& Marsolek, C. J. (2005). Does a causal relation exist between the functional hemispheric asymmetries of visual processing subsystems? Brain and Cognition, 59, 135-144.

Bar, M., Kassam, K. S., Ghuman, A. S., Boshyan, J., Schmid, A. M., Dale, A. M., et al. (2006). Top-down facilitation of visual recognition. Proceedings of the National Academy of Sciences, U.S.A., 103, 449-454.

Boles, D. B. (1998). Relationships among multiple task asymmetries: II. A large-sample factor analysis. Brain and Cognition, 36, 268-289.

Brem, S., Bucher, K., Halder, P., Summers, P., Dietrich, T., Martin, E., et al. (2006). Evidence for developmental changes in the visual word processing network beyond adolescence. Neuroimage, 29, 822-837.

Brysbaert, M. (1994). Interhemispheric transfer and the processing of foveally presented stimuli. Behavioural Brain Research, 64, 151-161.

Busey, T. A., \& Vanderkolk, J. R. (2005). Behavioral and electrophysiological evidence for configural processing in fingerprint experts. Vision Research, 45, 431-448.

Cantalupo, C., \& Hopkins, W. D. (2001). Asymmetric Broca's area in great apes. Nature, 414, 505.

Cohen, L., \& Dehaene, S. (2004). Specialization within the ventral stream: The case for the visual word form area. Neuroimage, 22, 466-476.

Cohen, L., Dehaene, S., Naccache, L., Lehericy, S., Dehaene-Lambertz, G., Henaff, M. A., et al. (2000). The visual word form area: Spatial and temporal characterization of an initial stage of reading in normal subjects and posterior split-brain patients. Brain, 123, 291-307.

Dehaene, S., Cohen, L., Sigman, M., \& Vinckier, F. (2005). The neural code for written words: A proposal. Trends in Cognitive Sciences, 9, 335-341.

Dehaene, S., Jobert, A., Naccache, L., Ciuciu, P., Poline, J. B., Le Bihan, D., et al. (2004). Letter binding and invariant recognition of masked words: Behavioral and neuroimaging evidence. Psychological Science, 15, 307-313.

Devlin, J. T., Jamison, H. L., Gonnerman, L. M., \& Matthews, P. M. (2006). The role of the posterior fusiform gyrus in reading. Journal of Cognitive Neuroscience, 18, 911-922.

Erdmann, B., \& Dodge, R. (1898). Psychologische Untersuchungen uber das Lesen, auf Experimenteller Grundlage. Halle: N. Niemeyer.

Frith, C. D., Friston, K. J., Liddle, P. F., \& Frackowiak, R. S. (1991). A PET study of word finding. Neuropsychologia, 29, 1137-1148.

Gauthier, I., Curran, T., Curby, K. M., \& Collins, D. (2003). Perceptual interference supports a non-modular account of face processing. Nature Neuroscience, 6, 428-432.

Gazzaniga, M. S. (1983). Right hemisphere language following commissurotomy: A twenty-year perspective. American Psychologist, 38, 527-537.

Gold, B. T., \& Rastle, K. (2007). Neural correlates of morphological decomposition during visual word recognition. Journal of Cognitive Neuroscience, 19, 1983-1993.

Grill-Spector, K., Sayres, R., \& Ress, D. (2006). High-resolution imaging reveals highly selective nonface clusters in the fusiform face area. Nature Neuroscience, 9, 1177-1185.

Gros, H., Doyon, B., Rioual, K., \& Celsis, P. (2002). Automatic grapheme processing in the left occipitotemporal cortex. NeuroReport, 13, 1021-1024.

Hagemann, D. (2004). Individual differences in anterior
EEG-Asymmetry: Methodological problems and solutions. Biological Psychology, 67, 157-182.

Hillis, A. E., Newhart, M., Heidler, J., Barker, P., Herskovits, E., \& Degaonkar, M. (2005). The roles of the "visual word form area" in reading. Neuroimage, 24, 548-559.

Hunter, Z. R., Brysbaert, M., \& Knecht, S. (2007). Foveal word reading requires interhemispheric communication. Journal of Cognitive Neuroscience, 19, 1373-1387.

Ishai, A., Ungerleider, L. G., Martin, A., Schouten, J. L., \& Haxby, J. V. (1999). Distributed representation of objects in the human ventral visual pathway. Proceedings of the National Academy of Sciences, U.S.A., 96, 9379-9384.

Knecht, S., Drager, B., Deppe, M., Bobe, L., Lohmann, H., Flöel, A., et al. (2000). Handedness and hemispheric language dominance in healthy humans. Brain, 123, 2512-2518.

Knecht, S., Drager, B., Floel, A., Lohmann, H., Breitenstein, C., Deppe, M., et al. (2001). Behavioural relevance of atypical language lateralization in healthy subjects. Brain, 124, 1657-1665.

Kosslyn, S. M. (1987). Seeing and imagining in the cerebral hemispheres: A computational approach. Psychological Review, 94, 148-175.

Law, S. K., Rohrbaugh, J. W., Adams, C. M., \& Eckardt, M. J. (1993). Improving spatial and temporal resolution in evoked EEG responses using surface Laplacians. Electroencephalography and Clinical Neurophysiology, 88, 309-322.

Malach, R., Levy, I., \& Hasson, U. (2002). The topography of high-order human object areas. Trends in Cognitive Sciences, 6, 176-184.

Maurer, U., Brem, S., Bucher, K., \& Brandeis, D. (2005). Emerging neurophysiological specialization for letter strings. Journal of Cognitive Neuroscience, 17, 1532-1552.

McCandliss, B. D., Cohen, L., \& Dehaene, S. (2003). The visual word form area: Expertise for reading in the fusiform gyrus. Trends in Cognitive Sciences, 7, 293-299.

Nazir, T. A., Ben-Boutayab, N., Decoppet, N., Deutsch, A., \& Frost, R. (2004). Reading habits, perceptual learning, and recognition of printed words. Brain and Language, 88, 294-311.

New, B., Pallier, C., Ferrand, L., \& Matos, R. (2001). Une base de données lexicales du français contemporain sur internet: Lexique. L'Année Psychologique, 101, 447-462.

Oldfield, R. C. (1971). The assessment and analysis of handedness: The Edinburgh Inventory. Neuropsychologia, 9, 97-113.

Perrin, F., Bertrand, O., \& Pernier, J. (1987). Scalp current density mapping: Value and estimation from potential data. IEEE Transactions on Bio-Medical Engineering, 34, 283-288

Petersen, S. E., Fox, P. T., Posner, M. I., Mintun, M., \& Raichle, M. E. (1988). Positron emission tomographic studies of the cortical anatomy of single-word processing. Nature, 331, 585-589.

Polk, T. A., Stallcup, M., Aguirre, G. K., Alsop, D. C., D'Esposito, M., Detre, J. A., et al. (2002). Neural specialization for letter recognition. Journal of Cognitive Neurosciences, 14, $145-159$.

Pujol, J., Deus, J., Losilla, J. M., \& Capdevila, A. (1999). Cerebral lateralization of language in normal left-handed people studied by functional MRI. Neurology, 52, 1038-1043.

Price, C. J., \& Devlin, J. T. (2003). The myth of the visual word form area. Neuroimage, 19, 473-481.

Price, C. J., \& Friston, K. J. (2005). Functional ontologies for cognition: The systematic definition of structure and function. Cognitive Neuropsychology, 22, 262-275.

Pulvermüller, F., Lutzenberger, W., \& Preissl, H. (1999). Nouns and verbs in the intact brain: Evidence from 
event-related potentials and high-frequency cortical responses. Cerebral Cortex, 9, 497-506.

Rowan, A., Liégeoisa, F., Vargha-Khadem, F., Gadian, D., Connelly, A., \& Baldeweg, T. (2004). Cortical lateralization during verb generation: A combined ERP and fMRI study. Neuroimage, 22, 665-675.

Sun, T., \& Walsh, C. A. (2006). Molecular approaches to brain asymmetry and handedness. Nature Reviews: Neuroscience, 7, 655-662.

Szaflarski, J. P., Binder, J. R., Possing, E. T., McKiernan, K. A., Ward, B. D., \& Hammeke, T. A. (2002). Language lateralization in left-handed and ambidextrous people: fMRI data. Neurology, 59, 238-244.

Tanaka, J. W., \& Curran, T. (2001). A neural basis for expert object recognition. Psychological Science, 12, 43-47.

Thiel, A., Herholz, K., von Stockhausen, H. M., LeyenPilgram, K., Pietrzyk, U., Kessler, J., et al. (1998). Localization of language-related cortex with 15O-labeled water PET in patients with gliomas. Neuroimage, 7 , 284-295.
Thomas, C., Altenmuller, E., Marckmann, G., Kahrs, J., \& Dichgans, J. (1997). Language processing in aphasia: Changes in lateralization patterns during recovery reflect cerebral plasticity in adults. Electroencephalography and Clinical Neurophysiology, 102, 86-97.

Vallortigara, G., \& Rogers, L. J. (2005). Survival with an asymmetrical brain: Advantages and disadvantages of cerebral lateralization. Behavioral and Brain Sciences, 28, 575-589; discussion 589-633.

Vigneau, M., Jobard, G., Mazoyer, B., \& Tzourio-Mazoyer, N. (2005). Word and non-word reading: What role for the visual word form area? Neuroimage, 27, 694-705.

Xue, G., Chen, C., Jin, Z., \& Dong, Q. (2006). Cerebral asymmetry in the fusiform areas predicted the efficiency of learning a new writing system. Journal of Cognitive Neuroscience, 18, 923-931.

Young, A. W., \& Ellis, A. W. (1985). Different methods of lexical access for words presented in the left and right visual hemifields. Brain and Language, 24, 326-358. 